vol. $21-n^{\circ} 1 \mid 2005$

Femmes, genre, migration et mobilités

\title{
Les filles naissent après les garçons
}

Représentations sociales des populations d'origine maghrébine en

France

Noria Boukhobza

\section{Q OpenEdition}

1 Journals

Édition électronique

URL : https://journals.openedition.org/remi/2333

DOI : 10.4000/remi.2333

ISSN : $1777-5418$

Éditeur

Université de Poitiers

Édition imprimée

Date de publication : 29 mai 2005

Pagination : 227-242

ISBN : 2-911627-39-3

ISSN : 0765-0752

Référence électronique

Noria Boukhobza, "Les filles naissent après les garçons 》, Revue européenne des migrations internationales [En ligne], vol. $21-\mathrm{n}^{\circ} 1$ | 2005, mis en ligne le 08 septembre 2008, consulté le 14 avril 2022. URL : http://journals.openedition.org/remi/2333; DOI : https://doi.org/10.4000/remi.2333

Ce document a été généré automatiquement le 14 avril 2022

(c) Université de Poitiers 


\title{
Les filles naissent après les garçons
}

\author{
Représentations sociales des populations d'origine maghrébine en \\ France
}

\author{
Noria Boukhobza
}

1 Il est désormais bien établi que les femmes migrantes furent longtemps oubliées de l'histoire, la migration ne pouvant se conjuguer dans les représentations sociales et dans les pratiques institutionnelles et publiques qu'au masculin (recensement des populations immigrées ne ventilant pas systématiquement les données par sexe, politiques publiques orientées vers les hommes migrants, etc.) (Morokvasic, 1983). La migration des femmes était dès lors envisagée comme seconde, connexe, et les femmes immigrées elles-mêmes n'étaient de fait qu'une minorité dans la minorité. Longtemps aussi, les femmes migrantes maghrébines ou d'origine maghrébine n'ont été envisagées que dans l'espace privé. Socialement et sociologiquement "invisibles ", elles ont été victimes de représentations stéréotypées auxquelles n'échappaient que quelques rares recherches de terrain dont certaines portant sur la transmission entre les générations (Andézian et Streiff-Fénart, 1978). Malgré la mise en visibilité des filles, dans le débat public - postérieure à celle des garçons supposés "poser problème » - cet article voudrait montrer combien les stéréotypes accumulés, qui perdurent encore largement, rendent difficile la perception d'une réalité sociale complexe. Il s'agira donc de s'interroger sur les représentations sociales concernant les femmes «issues de l'immigration " en tenant compte des différences sexuées dans différents espaces: espaces du quartier, du politique et de la citoyenneté, de la famille, de l'école. Comment les femmes d'origine maghrébine

- mères, filles, voire immigrées clandestines - sont-elles perçues, nommées dans ces espaces? Quelles attentes sociales suscitent-elles? Ces attentes et processus de catégorisation sont-ils réellement sexués? Il s'agira aussi, en filigrane, de rendre compte d'une démarche, ethnologique en l'occurrence, qui conduit le chercheur à pénétrer au sein des familles et des quartiers. Ma réflexion, dans un mouvement de vaet-vient constant entre la recherche et la pratique, repose sur des observations fondées sur une multiplicité de regards étayés par ma propre expérience de femme «issue de 
l'immigration", d'actions d'animation socioculturelle, et de ma fonction actuelle d'enseignante et de formatrice d'enseignants.

\section{Invisibilité, mise en visibilité : « les filles naissent après les garçons »}

2 Dès les débuts de mes recherches à Toulouse et Clermont-Ferrand, j'avais orienté mon questionnement sur les activités socioculturelles des filles dans les quartiers d'habitat social. Que font-elles dans ces quartiers? Quels espaces occupent-elles? De quelles origines sont-elles? Quelle est leur place au sein de leur fratrie? Ces questions restaient en suspens. Nous étions en 1987 et s'interroger sur les filles était une gageure. Ces dernières, discrètes, ne posaient aucun problème ou étaient tenues pour ne poser aucun problème et se situaient donc hors du débat public. Peu d'écrits, peu de travaux d'ailleurs les concernaient. La délinquance largement médiatisée des jeunes hommes dans les quartiers contribuait à les rendre invisibles (Gaspard, 1995). Des expressions comme les "jeunes des quartiers" ont longtemps fait irrémédiablement penser aux seuls garçons et, de surcroît, d'origine maghrébine. Les différentes interrogations centrées uniquement sur « les jeunes » ont montré, au fil des années, un visage coloré des quartiers, un visage « d'origine maghrébine » mais au masculin.

3 Les filles naissent après les garçons. Telle est la leçon que l'on pourrait tirer de l'observation de l'invisibilité désormais déclinante des filles dans les quartiers et des divers mouvements sociaux auxquels ont pris part les jeunes " issus de l'immigration ", qui ont été largement couverts par les médias et qui trouvent leur point d'ancrage dans ces espaces sociaux parfois dits sensibles (les quartiers). Revenons sur les faits. 1981 : l'on assiste à la première émeute urbaine aux Minguettes dans la banlieue de Lyon ${ }^{1}$. 1983-1984 : filles et garçons battent le pavé dans ce qui restera comme la Marche des beurs - (ou la marche de l'égalité) -, terme inventé pour désigner les jeunes issus de l'immigration maghrébine (le terme "beurette» n'apparaitra que plus tard). 1986-1987 : la manifestation et les débats polémiques à propos du Code de la nationalité ont révélé à l'opinion et à la classe politique françaises l'ampleur d'un phénomène social dans lequel certains voient désormais un défi à relever et d'autres une menace à conjurer. Ces faits sociaux - comme la marche de l'égalité - ont largement surpris l'opinion. Ces jeunes, « issus de l'immigration », considérés comme des délinquants et rejetés dans les cités ghettos, font la "une" de l'actualité en imposant leur propre réaction aux campagnes racistes. Moins silencieux et moins soumis que leurs parents, ces enfants se révoltent. Ils se donnent les moyens d'agir collectivement en se fixant un objectif audacieux: ne pas rester dans l'ombre et le mépris comme la première génération, mais acquérir un espace comme citoyens français à part entière. On parlera d'un sursaut de citoyenneté de ces jeunes.

4 Même si des images d'époque montrent des filles, on gardera le souvenir d'un mouvement masculin, d'autant que ce sursaut réconforte après la médiatisation des violences perpétrées dans les quartiers, des faits divers ayant contribué à la stigmatisation de ces jeunes garçons. Peut-être a-t-on voulu voir un mouvement masculin dans un contexte où ce sont les garçons qui sont supposés poser problème, montrés du doigt comme fauteurs de trouble? Il reste que des femmes et jeunes filles ayant participé à ce mouvement, un peu laissées pour compte du battage médiatique, se sont engagées dans le mouvement associatif. Tout au long des années quatre-vingt, 
«insécurité, politisation de la question de l'immigration, crise dans les banlieues, débats sur le code de la nationalité et croissance du chômage sont associés" (Blanchard et al., 2003) à la présence maghrébine. Or ces thèmes se déclinent mieux au masculin. Années quatre-vingt-dix, les émeutes reprennent, les quartiers sont toujours stigmatisés par les médias et perçus au masculin. Années 2000: on assiste à la médiatisation croissante des violences faites aux femmes dans les quartiers. 2003 : la "Marche des femmes contre les ghettos et pour l'égalité " (février/mars) donne naissance au mouvement ni putes ni soumises (mouvement « mixte et populaire » comme il se présente lui-même). Il aura fallu attendre vingt ans pour qu'une marche des femmes issues des quartiers ou des cités mette enfin les filles sous le feu des projecteurs. Tout se passe comme si les femmes, "en marche », revendiquant l'égalité naissaient après-coup. Notons que les jeunes filles maghrébines sont peu nombreuses à adhérer à ce mouvement (tranche d'âge entre 18 et 30 ans) auquel leurs mères sont plus sensibles, peut-être parce que, contrairement à leurs filles, elles sont dans une parole autorisée ? (Guenif-Souilamas et Macé, 2004).

5 La recherche sociale a elle-même longtemps tenu dans l'invisibilité les filles dans les quartiers. Les terrains de recherche ont en effet été surdéterminés au masculin.

\section{La surdétermination des terrains de recherche au masculin}

6 À propos de quartiers d'habitat social à Toulouse, deux ethnologues soulignent justement :

«C'est un terrain saturé de chercheurs, d'étudiants, de travailleurs sociaux qui cherchent aussi, de faiseurs de projet... Il est traversé par de nombreux discours, c'est un véritable laboratoire social en ébullition [...]. Il ne faut pas oublier non plus, en ce qui concerne le Mirail, que l'université voisine, Toulouse-Le-Mirail, fournit en quelque sorte au milieu ses propres intellectuels et intervenants, repérés comme partenaires et engagés dans de nombreuses opérations culturelles dont le quartier est le terrain ressource. L'opération Banlieue-Université contribue encore à déverser sur le quartier des équipes, étudiants et enseignants, avec un programme de recherche-action » (Zoïa et Mignard, $1994: 15$ ).

7 Comment alors, aujourd'hui, travailler sur un terrain déjà mille fois arpenté ayant fourni matière à des discours surdéterminés et à des réponses souvent toutes faites ?

On interroge, ainsi, depuis plus de quinze ans souvent les mêmes personnes, une majorité d'hommes, repérés dans les structures associatives, ayant endossé plusieurs images précédemment évoquées : celle du délinquant, du beur intégré ou du grandfrère des années quatre-vingt, puis celle du médiateur incontournable, responsable d'une association, pour finir. Il faut rappeler que les objets de recherche axés sur les enjeux de la nationalité, les relations de la population d'origine maghrébine avec la commune, ou la délinquance des jeunes n'abordent pas ou prou les rapports sociaux entre hommes et femmes - hormis précisément ceux qui portent sur les violences sexuelles dans les quartiers - ou se désintéressent de l'entrée « femme ».

Nombre de chercheurs, ceux qui se nourrissent d'entretiens effectués auprès de jeunes, de femmes rencontrées dans des lieux déjà structurés, banalisés, comme l'école, les centres sociaux, ont pour leur part suscité une parole pré-construite et souvent répétitive. De façon lapidaire, lorsqu'elles sont étudiées pour elles-mêmes, les filles sont 
présentées de deux manières antinomiques ou extrêmes : on les dépeint tantôt comme des femmes émancipées, vecteurs de changement social tantôt comme des femmes entièrement soumises à la domination masculine.

En opposition à cette dernière image, des travaux qui sont principalement le fait de chercheuses « issues de l'immigration » et de femmes venues des pays du Maghreb pour effectuer en France leurs études supérieures ont permis de mettre en lumière les stratégies des femmes dans leur processus migratoire ou leur parcours de vie. Ces travaux malgré leur intérêt - mémoires et thèses de doctorats - ont cependant une faible visibilité, sont peu cités dans les bibliographies d'ouvrages et d'articles ${ }^{2}$ : autant de documents universitaires explorant des aspects de la vie des familles «issues de l'immigration » qui restent inexploités La plupart de ces femmes n'ont pas emprunté le " parcours fléché » ou idéal de la réussite universitaire qui conduit au poste de Maître de Conférences. Un oubli, une relégation, qui contribuent certainement à notre déficit de mémoire. L'invisibilité des recherches relatives aux femmes migrantes émanant de chercheuses "issues de l'immigration" ne fait que souligner la relation étroite unissant le chercheur et son objet de recherche. D'une certaine manière le chercheur hérite des représentations sociales liées à son sujet (Sayad, 1992).

\section{Figures imposées}

11 Les femmes qui viennent des mêmes quartiers que les hommes héritent-elles des mêmes figures? Celles qui sont repérées, et ceci vers les années quatre-vingt-dix seulement, occupent bénévolement des fonctions d'animatrices : c'est donc la figure de la «médiatrice culturelle » qui prime. Quant aux plus jeunes, elles héritent d'une image plus collective et construite de l'extérieur soit par les médias soit par les acteurs sociaux du système éducatif, il s'agit d'abord de l'image de la fille qui réussit sa scolarité, puis de la beurette intégrée (Assouline, 1992).

Une autre figure, bien distincte, émerge pourtant dans l'espace social. Les premières filles voilées, dans les années quatre-vingt-dix, font tache dans le paysage républicain français. Les "filles voilées", surtout les adolescentes, deviennent le catalyseur de positions contradictoires y compris parmi les féministes. Leur forte médiatisation masque du même coup les « filles échevelées ", qui n'adhèrent pas au choix du voile.

Douze ans après, de nouvelles figures s'imposent: celle de la victime des "tournantes $»^{3}$ des années 2000 au premier plan, celle de la musulmane "non intégrée ", ou encore de la "républicaine» qui se cache sous la bannière du mouvement «ni putes, ni soumises». Au sein des populations de même origine nationale, celles qui ont perdu leur virginité et principalement celles qui ont subi des violences sexuelles (viols collectifs) dans les quartiers, sont perçues comme souillées, elles tombent dans la disgrâce, héritant d'une image négative. L'honneur perdu les ravale au rang de "filles perdues", de "filles des rues» (Boukhobza, 2002). Les autres tendent tantôt à être perçues comme des victimes - et victimisées - par les médias, les travailleurs sociaux, l'opinion publique, tantôt comme des vecteurs de changement dès qu'elles défendent dans l'espace public les valeurs de la République. Sur ce terrain miné comment donc se libérer du discours tenu par les travailleurs sociaux et les promoteurs des politiques publiques sur l'intégration de cette population?

14 N'est-ce pas le fait de regarder «les quartiers" à travers la seule grille de lecture des politiques publiques qui fait courir le risque de reproduire des idées reçues? Les 
représentations réductrices concernant les familles migrantes en sont un bon exemple. Ces représentations des acteurs sociaux ne traversent-elles pas, en effet, tous les espaces y compris celui de la recherche en sciences sociales?

\section{Des familles stigmatisées}

15 «Les filles naissent après les garçons ", disions-nous. Comment ne pas voir dans cette apparition tardive des filles d'origine maghrébine dans les écrits, les médias, les recherches, le pendant d'un biais, celui qui a voulu qu'on accorde une place prépondérante aux garçons comparativement à leurs sœurs au sein des familles? Les recherches relatives aux enfants «issus de l'immigration» ont remodelé symboliquement les familles. En questionnant d'abord la place des frères et quelques années plus tard celle des sœurs, elles ont souvent postulé que toutes les familles étaient composées de la même façon : les aînés sont uniquement des garçons, les sœurs sont toujours présentées comme des cadettes. Cette reconfiguration des familles permet d'opérer une hiérarchisation du masculin et du féminin, et d'introduire une dimension de rapport de force entre les aînés masculins et les cadettes féminines. En désignant d'abord les frères, les sœurs sont ramenées, malgré elles, à un statut de mineures. Modéliser les familles, en mettant les filles au second plan, revenait à figer les fratries et surtout à ne pas s'interroger sur les relations, les tensions et les pacifications qui de fait s'opèrent en leur sein par le biais de négociations permanentes.

Peu d'études ont en effet questionné les rapports sociaux de sexe, les rapports de domination ou les négociations entre hommes et femmes au sein des familles immigrées. Les représentations actuelles tendent à faire émerger dans l'espace public des idées reçues laissant croire que les familles d'origine maghrébine seraient les mêmes sur tout le territoire national. Parce qu'elles sont considérées comme situées d'emblée dans un espace social donné et stigmatisé, celui "des quartiers dits sensibles ", elles apparaissent immuables dans le temps, comme si elles n'étaient pas traversées par les différents changements qui s'opèrent dans toutes les familles en France: séparations, divorces, diminution du nombre d'enfant, familles monoparentales, accès des femmes au travail (Boukhobza, 2001). L'image d'une famille nombreuse constituée principalement d'un grand frère, d'une sœur aînée aidant la famille, d'un père absent, reste encore dominante dans les recherches et les discours. Cette configuration familiale est le fruit de plusieurs facteurs : les politiques publiques, les médias, certaines recherches en sciences humaines, les faits historiques (et leur représentation) hérités du colonialisme. Par exemple, l'idée selon laquelle l'homme est juge, chef de famille, et la femme soumise à l'autorité du mari est une donnée non interrogée, prise comme allant de soi. La confrontation entre époux est largement ignorée. Dans cette imagerie, les mères sont perçues comme les gardiennes de la tradition ou les agents de transmission de la «culture familiale» qui passe essentiellement par elles. Elles ne peuvent être vues et étudiées que dans l'espace privé. Et l'on peut légitimement se demander si, la longue invisibilité des filles dans certains espaces sociaux n'est pas en grande partie le résultat de l'invisibilité de leurs mères en tant que migrantes sur la scène sociale? (Gaspard, 1995). 


\section{Hommes clandestins, femmes invisibles}

17 Concernant les flux migratoires plus récents et se trouvant en situation d'irrégularité sur le territoire français, les terminologies employées pour décrire les immigrées non maghrébines sont sexuées: on parle de "clandestins", jamais de "clandestines", comme si ceux/celles qui traversent les frontières sans titre de séjour n'étaient que des hommes. Il faudra attendre des travaux portant sur les mouvements sociaux au sein et autour de ces groupes pour que les termes employés soient féminisés. En effet, l'utilisation du terme "sans-papières » auquel se réfère Catherine Quiminal constitue une façon de rendre compte de leur trajectoire (Quiminal, 2000). Les terminologies séparent les hommes des femmes. Ces mots rendent invisible la venue des femmes. Les hommes traversent les frontières, pas les femmes apparemment, et pourtant nous les retrouvons ensuite, sous la plume de journalistes, d'observateurs ou de chercheurs, sous la coupe de «réseaux criminels» ou de proxénètes. Elles sont, pour accuser le trait, prostituées ou victimes de trafics de femmes, au mieux employées domestiques. C'est une vision de victime qui domine : rien n'est dit du caractère parfois volontaire de la venue de ces femmes. Et lorsqu'on évalue leur parcours, c'est généralement pour dire qu'elles ont été trompées sur les véritables motivations et finalités de leur migration, leur passage des frontières, leur voyage : l'éventuelle maîtrise par ces femmes de leur projet migratoire est occultée.

Qu'en est-il des clandestines originaires d'Afrique du Nord? On ne retrouve pas, à propos de ces dernières, les images accolées aux autres clandestines déjà mentionnées (prostituées, victimes de la traite des femmes...). En outre, pas plus que les jeunes filles maghrébines et autres dans les quartiers à une certaine époque, les femmes clandestines originaires d'Afrique du Nord ne posent problème. On ne les voit, de fait, pratiquement pas: elles sont à peine aperçues, à l'occasion, dans les médias. Mais contrairement aux autres femmes immigrées non clandestines, elles ne relèvent pas d'une problématique du "quartier » et n'existent que comme catégorie générale dans un espace plus large : le territoire national et international. Les questionnements les concernant sont aussi en lien avec le statut particulier qu'occupent les femmes dans ce contexte migratoire. Femmes «rejoignant», épouses, étudiantes, célibataires: plusieurs profils migratoires, plusieurs stratégies... Or, lorsque ces femmes sont issues de pays d'Afrique au sens large, elles sont d'abord perçues sous l'angle du regroupement familial, comme des épouses ou des futures épouses. Ces dernières rencontrent des difficultés dans l'obtention de titres de séjour et sont, au même titre que leurs homologues en situation régulière et présentes sur le territoire national depuis de longues années, soumises aux statuts personnels, un droit du pays d'origine qui s'applique sur le territoire national (Gaspard, 1994). Les institutions portent alors un regard sexué sur le parcours de ces migrants et migrantes. D'après des observations menées dans le cadre de mes recherches (Boukhobza, 2002) des femmes ayant migré au titre du regroupement familial se sont entendu rappeler au moment de la remise de leur certificat d'hébergement à la préfecture qu'elles sont interdites de travail. Interdiction réelle, formulée oralement en présence du conjoint qui renforce, de fait, l'inégalité sexuée. 


\section{Des femmes identifiées par leur sexe ; des hommes par leur origine « ethnique-nationale »?}

19 situation régulière, pendant longtemps, les jeunes filles d'origine maghrébine n'ont été
visibles que dans l'espace de l'école: diverses études soulignent ainsi la meilleure réussite scolaire des filles comparativement aux garçons de même origine nationale (Hassini, 1997). Mais elles restaient invisibles, tout comme leurs mères, donc, dans d'autres sphères de la vie publique - emploi, politique, engagements associatifs, etc. Puis, depuis peu se développent les études sur la place des filles dans les quartiers. Souvent lorsque les femmes investissent d'autres espaces où l'on ne pense pas les trouver, ce n'est plus tant à travers leur origine nationale ou leur assignation à une origine ethnique qu'elles sont perçues, classées, catégorisées, nommées, mais à partir de leur identité de genre. C'est ici le fait d'être une femme qui prime sur les autres formes d'appartenances. Ainsi, un diagnostic portant sur le chômage des jeunes (où l'on s'attend à trouver de nombreuses personnes d'origine immigrée) identifiera, repérera certainement une fille qui s'adresse aux agences d'intérim, aux missions locales parce qu'elle est une femme "issue de l'immigration» sans emploi, quoique qualifiée. Comptabilisée comme personne en difficulté, elle suscitera l'intérêt des travailleurs sociaux. En revanche, une femme d'«origine immigrée" occupant un poste à responsabilité, sera repérée uniquement en tant que femme par les chercheurs, à l'occasion d'une étude sur la présence des femmes dans des secteurs d'activité masculins. Cette identification à partir du sexe prime sur l'origine ethnique ou nationale de la personne. L'origine ethnique n'est donc plus un facteur pertinent pour expliquer la présence de cette personne dans cet espace. Les femmes d'«origine immigrée » occupant des emplois dans des secteurs d'activité peu ouverts aux femmes ou accédant à des postes de responsabilité seront de la sorte une nouvelle fois rendues invisibles.

Un autre exemple vient illustrer ce propos. Sur notre terrain en Midi-Pyrénées, nous avons eu l'occasion d'observer les différences entre hommes et femmes en matière d'accès au marché du travail (Boukhobza, Delavaut, Hermann, 2002) ${ }^{4}$. Quand une femme d'" origine étrangère " possédant un niveau Bac +5 opte pour un emploi, elle est recrutée sur la base de ses compétences professionnelles. Cependant, elle rencontre, à l'instar de toutes les autres femmes, des difficultés en termes d'évolution de carrière. Un homme de même origine nationale, engagé dans la même démarche d'insertion au niveau d'un poste de cadre, sera recruté à un poste inférieur. Certains employeurs avoueront d'ailleurs qu'ils ne souhaitent pas donner la direction à un jeune d'origine maghrébine, afin d'éviter des problèmes avec l'ensemble du personnel qui ne souhaite pas être placé sous ses ordres ${ }^{5}$.

21 Les hommes immigrés ou d'« origine immigrée » occupant des postes à responsabilité seront en revanche identifiés en fonction de leur origine ethnique, nationale, culturelle, religieuse. Outre les employeurs avouant ne pas recruter de garçons d'origine maghrébine, nous assistons à la médiatisation de cas de jeunes hommes maghrébins, qualifiés, titulaires de diplômes et ne trouvant pas d'emploi. On entend souvent dire à leur propos qu'ils ne sauraient constituer des modèles pour les autres jeunes des cités. 
Ces exemples supposés ou allégués de discrimination semblent, dans les discours, moins toucher les filles. Cet état de fait soulève des questions : les garçons maghrébins rencontrent-ils effectivement plus de difficultés à s'insérer sur le marché de l'emploi que leurs homologues féminines? Sont-ils réellement plus souvent sujets aux discriminations? Ces différences sont-elles liées à la spécificité des secteurs d'activité ou aux postes occupés par ces jeunes hommes? Ou bien le discours sur la discrimination des hommes maghrébins n'est-il pas, lui aussi, imputable pour partie à un regard biaisé ? Celui qui détecte plus facilement l'origine nationale des hommes dans l'accès aux emplois qualifiés? Ou au fait que les discriminations des jeunes femmes de même origine ethnique ou nationale sur le marché de l'emploi sont masquées par leur appartenance sexuée ? En effet, la discrimination des femmes

- dans l'accès à des postes de responsabilité par exemple - si elle ne prend pas la forme d'une distinction à partir de l'origine ethnique ou nationale, peut emprunter des chemins détournés, s'appuyer sur le nombre d'enfants, bref s'ancrer sur l'appartenance au sexe féminin, autrement dit, aux prétendues responsabilités familiales inhérentes aux femmes. À nouveau, chez les femmes, l'importance de la dimension sexuée semble l'emporter sur les autres formes d'appartenance.

Outre une plus forte invisibilité de l'« origine immigrée » des femmes dans les emplois qualifiés comparativement à leurs homologues masculins, il est possible de noter d'autres différences sexuées relatives, cette fois-ci, aux processus consistant à nommer les individus. Dans certains emplois comme la vente ou la coiffure, les employées sont rebaptisées. Sabria devient "Sabrina », Malika se transforme en " Nadia », etc., afin de rendre leurs prénoms "plus acceptables et moins marqués culturellement». Les femmes ne mesurent pas toujours la portée du travestissement de leur prénom, croyant naïvement qu'il s'agit simplement d'être dans la mode du moment. Les hommes qui travaillent dans le bâtiment ou dans les usines quel que soit leur prénom sont souvent prénommés «Momo », diminutif de Mohammed. Une différence dans les pratiques de nomination qui renvoient à des modes d'identification distincts des hommes et des femmes immigrés ou «issus de l'immigration" dans la sphère professionnelle: référence expresse à l'identité culturelle chez les hommes, gommage des traits culturels pour les femmes. Peut-être faut-il y voir, en partie, le résultat du mode d'inscription de ces femmes dans des emplois de service, au contact d'une clientèle, où il sied de faire "bonne figure", dans un contexte où la présentation de soi joue directement sur le standing social et l'image de marque de l'entité qui les emploie (salons de coiffure, commerces, etc.)?

\section{La « médiation culturelle » : pour les femmes, un engagement ; pour les hommes, un métier}

D'autres différences sexuées peuvent encore être relevées. Le rapport au travail salarié analysé à travers l'exemple de la professionnalisation de ce que l'on appelle la «médiation culturelle " mérite d'être mentionné. Dans les années 1980 apparaissent conjointement « les grands frères » et les médiateurs culturels. Dans le cadre de l'IUT d'animation socioculturelle de Tours, pour prendre un exemple observé directement, de jeunes hommes sont formés à occuper un rôle d'interface, la formation est sanctionnée par l'obtention d'un diplôme permettant l'accès à un emploi dans le cadre d'une convention collective. Le terme d'« intermédiaires culturels» est transposé aux 
populations algériennes par Thierry Fabre "pour désigner tous ceux qui par leurs pratiques parviennent à ouvrir des passages, à construire des médiations entre deux ensembles culturels » (Pottier, 1993). Les femmes de même origine jouaient ce rôle dans les années quatre-vingt. Mais leurs pratiques étaient d'abord un engagement individuel qui pouvait s'inscrire ou non par la suite dans un cadre associatif. Elles faisaient de la traduction, jouaient les intermédiaires au sein de leur entourage, puis entre le voisinage et les administrations. La source de leur savoir-faire résidait dans les difficultés qu'elles-mêmes avaient affrontées dans leur vie quotidienne.

On peut rappeler une étude conduite par Catherine Delcroix en 1993 sur « le rôle et la perspective des médiateurs interculturels » qui a inscrit les femmes migrantes ou d'origine migrante dans un espace déterminé, celui du quartier. Repérées comme des femmes relais, ces dernières devenaient, malgré elles, des vecteurs du changement. Leur position d'aînée dans leur fratrie, leur vécu dans le quartier et leur histoire avec les habitants, les plaçaient dans une interface entre l'institution et la population, en créant ainsi un lieu intermédiaire de rencontre.

À Clermont-Ferrand, dans le cadre d'une formation que j'ai coordonnée visant à identifier des personnes susceptibles de tenir le rôle de "femmes relais", les critères d'admissibilité fixés par la Délégation régionale du droit des femmes épousaient le stéréotype appliqué aux femmes maghrébines: absence de travail de femmes d'« origine étrangère » se trouvant en situation de précarité, ayant du temps à investir dans les mouvements associatifs, femmes mariées de préférence car dépositaires de la bonne image, de la bonne tenue dans le quartier au regard des familles. Ces femmes, tout en servant d'interface entre les institutions et les familles, ne devaient pas empiéter sur les prérogatives de l'institution, elles étaient de simples associées dans le cadre défini par celle-ci. Cela signifiait l'exclusion des femmes détentrices de postes à responsabilité dans les associations, dotées de relations étendues au sein du milieu associatif au profit des « femmes de maison ».

27 À la formation professionnalisante des hommes s'oppose ainsi celle des femmes, relevant du bénévolat, sans perspective d'insertion sur le marché de l'emploi. Peut-être faut-il y voir en partie la résistance des travailleurs sociaux qui pouvaient voir en ces femmes de potentielles rivales. En effet, ces « femmes-relais ", " agents de proximité », " médiatrices », etc., selon les appellations, dénominations qui leur sont données ne se limitent pas à recevoir les femmes du quartier au sein des diverses structures qui y sont implantées, elles ont la possibilité de pénétrer dans les espaces privés des familles, ce que ne peuvent faire les travailleurs sociaux sauf à y être conviés.

28 Cependant, dans le cadre des politiques de la ville, on assiste depuis quelques années à la création d'emploi-jeunes liés à la médiation culturelle dans les écoles proches des quartiers. Les filles ayant suivi des formations de "médiatrices culturelles» ont souhaité intégrer ces postes. Or, dans les zones d'éducation prioritaire (ZEP), on a préféré recruter des garçons supposés avoir «le physique " adéquat permettant de résoudre les problèmes de violence au collège. Ce sont donc des garçons, et non des filles, qui ont obtenu des postes d'animateurs au sein des collèges sous l'appellation de "médiateurs culturels ". Ici à nouveau se devine une discrimination sexuée. Au-delà de ce constat, il faut noter que l'introduction de cette fonction au sein du système éducatif a fait naître de nouvelles figures de médiateurs qui ne manquent pas de poser problème et qui rendent compte d'une «division ethnique du travail social» : « un recrutement de jeunes formés sur la base de leurs compétences endogènes ou indigènes, et non sur 
la base de qualifications certifiées par des diplômes semble s'accommoder de cette division ethnique du travail social et [il semble qu'] après tout, l'ethnicisation des rapports sociaux est désormais une donnée évidente des relations dans les quartiers " (Billion, 2001 : 116).

29 Assignés malgré eux à des rôles semblables, des enseignants d'origine maghrébine héritent, à leur corps défendant, de l'image de médiateur, de personne-ressource dans le cadre de l'exercice même de leur profession. De manière explicite, on les sollicite en fonction de leur origine.

\section{Des enseignant-e-s sollicité-e-s pour leur origine culturelle}

30 Longtemps, les jeunes « issus de l'immigration » n'ont pas été recrutés par l'Éducation nationale. Des travaux n'ont pas manqué de souligner que l'accès de ces jeunes aux postes d'enseignants les érigerait en modèle pour les élèves d'origine étrangère ou issus de minorités ethniques et serait à même d'élever leurs résultats scolaires (Assouline, 1992). Cette lacune est aujourd'hui comblée. Ils accèdent de plus en plus nombreux à ces fonctions. En quoi seraient-ils différents des autres? Ma fonction de formatrice à l'IUFM m'a conduite à rencontrer et à débattre avec de nombreux enseignants d'origine maghrébine mais pas uniquement. Cette position m'a amenée aussi à intervenir sur la relation enseignant/élèves et à aborder la question de la mixité : mixité sociale, culturelle, et de sexe. Il ressort des débats entre enseignants qu'ils sollicitent fréquemment leurs confrères "issus de l'immigration" en fonction de leur connaissance supposée du public concerné (les élèves d'« origine immigrée »). Ces enseignants, renvoyés à leur origine culturelle, endossent le rôle de médiateurs/médiatrices, celui d'intermédiaires, ou de personnes supposées aptes à résoudre des conflits.

31 Ces jeunes enseignants doivent s'approprier, souvent malgré eux, l'histoire des jeunes «issus de l'immigration» et l'histoire familiale que l'on croit commune à tous. Les attentes - et les réactions - sont cependant différentes selon le sexe des enseignants concernés. Dans les lycées professionnels, nous trouvons des hommes, généralement titulaires du CAPET $^{6}$, enseignant dans des classes massivement composées d'élèves d'origine maghrébine. Les femmes, titulaires du $\mathrm{CAPES}^{7}$, enseignent l'anglais, les lettres, etc. dans les collèges et les lycées d'enseignement général à un public plus diversifié en termes d'origine nationale. Les premiers, logés à la même enseigne que les autres enseignants (non « issus de l'immigration ») quand il s'agit d' " amadouer » ou de «tenir» leur classe dans les lycées professionnels semblent moins sollicités que les secondes dans les lycées généraux. La composition même des classes (en termes d'origine ethnique) influence donc les sollicitations qui diffèrent selon les sexes (les femmes sont plus sollicitées que les hommes). Les enseignants masculins des filières hôtelières, par exemple, où l'on trouve peu d'élèves d'origine maghrébine ont affirmé revêtir de fait cette fonction informelle de médiateur. En outre, les femmes, plus que les hommes, manifestent leur sentiment de malaise face à cette sollicitation, arguant d'un parcours familial et social distinct des élèves auxquels on les assimile a priori. Supposer que ces personnes détiennent des réponses adaptées de par leur «origine nationale " ou «ethnique " crée un malaise. Cette sollicitation plus fréquente des femmes n'est en outre certainement pas étrangère à l'idée d'une propension naturelle 
des femmes à servir d'intermédiaires, de médiatrices afin de résoudre des conflits. L'une des images attribuées aux jeunes femmes "issues de l'immigration » (au double sens de "provenant» et de "sorties»), celle de femmes émancipées, porteuses de changement social est certainement mobilisée dans ce contexte. L'injonction est paradoxale : par leur origine, ces femmes sont supposées être dans une proximité avec les élèves "issus de l'immigration ", et ce indépendamment de leur parcours, mais on attend d'elles, aussi, les solutions ou réponses que le corps enseignant et l'encadrement ne semblent pas à même de proposer. Il en est autrement pour les enseignants hommes de même origine dans les lycées généraux: ils disent ne pas être sollicités car, selon eux, les élèves de même origine qu'eux sont peu nombreux dans les filières représentées au sein desquelles ils effectuent un parcours de réussite. D'autres observations viennent conforter cette idée d'un renvoi des femmes à leur histoire familiale et, plus généralement, à celle du quartier.

\section{Mémoires de filles, histoires de quartiers}

Pour apprécier la réification de l'image des femmes issues de l'immigration maghrébine, leur renvoi à leur famille et leur assignation à l'espace du quartier, je prendrai pour exemple les observations découlant d'une recherche/action menée dans un quartier de Toulouse et intitulée " Mémoires de filles, histoires de quartier " ${ }^{8}$. Dans le cadre d'un groupe de parole, l'occasion a été donnée à des jeunes filles de toutes origines de débattre sur des thèmes aussi divers que le rapport mère-fille, la violence dans les quartiers, la généalogie, le rôle des récits oraux dans la construction du masculin et du féminin, et enfin la question du mariage (Boukhobza, 2005). Il s'agit d'une action orientée vers des jeunes femmes, qui progresse par maturation lente, peu «visible» par certains aspects au regard des critères institutionnels habituels. C'est une action inscrite dans la durée, dans la fidélité de la relation établie avec les filles, dans une confiance mutuelle construite au fil du temps. Cette caractéristique est parfois mal comprise à l'extérieur car sans résultats immédiats, tangibles, permettant de justifier un financement institutionnel. Le groupe a donc choisi de sortir de sa phase réflexive pour s'engager sur la scène sociale par ses propres actions; d'abord en participant à des festivals de cinéma, puis en créant des rencontres/débats sur des sujets qui lui tiennent à cœur.

Prenons l'exemple de la participation du groupe au festival de film international de femmes à Créteil. L'association (Intermed') a exigé qu'il n'y ait pas d'éducateur accompagnant afin d'éviter que les filles ne soient repérées comme venant «d'un quartier » ou ayant « des problèmes », afin qu'elles ne soient pas stigmatisées d'emblée devant le public du festival. Ce choix va créer une rupture entre les éducateurs et les membres d'Intermed'. Des négociations font émerger la problématique suivante: «les filles appartiennent d'abord au quartier». Pour cette raison, elles doivent être accompagnées de travailleurs sociaux intervenant dans «leur » quartier. Les échanges furent difficiles et épineux, il nous a fallu rappeler qu'elles étaient majeures et qu'elles ne ressortaient d'aucun dispositif particulier.

La première fois, les filles reconnaissent avoir mal évalué l'intérêt de se rendre à Créteil. Certaines ne voient pas le moment clef que représente la venue au festival dans le parcours du groupe. Venant pour certaines de familles assez strictes, elles voulaient d'abord satisfaire à tout prix un désir personnel. Sur le temps court d'un bref séjour au 
festival, tout tourne autour du projet de sortir en boîte de nuit, de "s'éclater sans avoir les parents sur le dos ». C'est aussi un moment où des tensions éclatent au sein du groupe. Des filles expriment leur frustration, leur colère même devant l'attitude de certaines autres qui sont accusées d'avoir "gâché l'ambiance ». Cette crise provoque $a$ posteriori un déclic sur le sens et l'intérêt de l'expérience du groupe de parole. Une dynamique interne se construit peu à peu, soudant les participantes dans l'expérience partagée.

Après le premier voyage à Créteil, les éducateurs pensaient qu'il n'y aurait pas de "retour ", comme s'il ne s'était agi que d'une escapade ludique. Ils auraient voulu que le groupe atteste du contraire en faisant rapidement un bilan. Mais le contexte restait compliqué du fait des suites du conflit. Répondre immédiatement à cette sollicitation serait revenu à prendre le risque de faire éclater le groupe, alors très fragilisé. Il a fallu du temps, près de deux mois, pour laisser se décanter et digérer l'épisode avant de pouvoir organiser à nouveau une rencontre collective. Mais les éducateurs se sont sentis trahis par cet apparent temps mort dans l'action.

En fait le «retour » a eu lieu un an après, à l'occasion d'un débat public organisé au cinéma Utopia à Toulouse, une occasion pour le groupe d'inscrire leur action dans la ville. Les filles ont choisi comme support un film chinois sur l'enfance « La môme singe ». À la fin de la projection, plusieurs questions ont été posées par des travailleurs sociaux : d'abord la raison du choix d'un tel film, comme s'il était incompréhensible de ne pas avoir préféré plutôt une œuvre qui fasse référence à la culture d'origine ou au quartier ; la question ensuite était formulée ainsi : « et vos mères?». Pourquoi l'action n'est-elle que du côté des filles et pas aussi du côté des mères? Comme si le gain d'autonomie des filles renvoyait à l'oubli des mères, à la famille. Enfin la dernière question portait sur les frères : "sont-ils d'accord pour que vous vous émancipiez de la sorte? ". C'est un moyen de leur dire de ne jamais oublier d'emmener dans leurs bagages leurs parents, leurs frères et l'histoire entière de leur quartier. Selon mon interprétation, il s'agit finalement de culpabiliser les filles quand elles prennent trop de libertés et surtout de les inscrire dans " une filiation territorialisée ».

37 À la suite de cette confrontation, les membres du groupe prennent conscience qu'il faut être capable d'argumenter pour aller à l'encontre des représentations sociales et des stéréotypes en circulation. Le problème de l'assignation à un quartier émerge alors dans les débats, les filles sont repérées comme venant d'un quartier «dit difficile»: d'abord à l'école, dans les demandes d'emploi d'été, puis dans la recherche d'un emploi plus stable. Dans les questions, qui leur sont posées disent-elles, il apparaît que les filles endossent toujours l'histoire de la cité alors même qu'elles ne construisent pas cette histoire.

38 Après ce long travail, les filles ont pu, d'une part, reconstruire et se réapproprier une histoire, dont elles sont devenues les actrices et, d'autre part, intervenir aujourd'hui à la demande des professionnels du système éducatif dans des écoles situées en ZEP. Notre démarche sur le terrain a consisté d'abord à ouvrir un espace de débat et de participation assez libre pour que chaque femme puisse s'y glisser, se l'approprier et le recomposer à sa manière.

39 Ainsi peut s'exprimer une parole plurielle, attentive à la mémoire féminine du destin migrant quelle que soit la génération considérée. Il s'agit de reconnaître la grande diversité des femmes et des filles "issues de l'immigration ». La circulation dans les espaces collectifs, les itinéraires professionnels comme les parcours de vie ne sont pas 
semblables. Pour chacune, la structure familiale, la parentèle, tout comme l'éducation reçue, dessinent une configuration bien particulière. Le statut familial et sexuel est bien sûr d'une prime importance: parmi ces jeunes femmes, certaines sont encore "jeunes filles", d'autres sont déjà mères. Mais il ne faut pas non plus oublier les relations différemment vécues au sein du quartier, ni les différences qui s'opèrent en fonction de l'activité de chacune : lycéenne, étudiante, en recherche d'emploi ou en activité, engagées ou non dans une association... Il s'agit dès lors de prendre en compte les trajectoires professionnelles et le vécu individuel, les stratégies, les compétences, les choix, les négociations qui caractérisent chacune de ces femmes en fonction de sa propre subjectivité et qui induisent des mouvements constants d'adaptation, de composition et de recomposition.

\section{Conclusion}

L'aller-retour entre la recherche et l'action m'a permis d'appréhender, dans divers espaces sociaux, au plus près du terrain, diverses manières de regarder, nommer, catégoriser les femmes et les hommes issus de l'immigration maghrébine. Ces processus sont différenciés selon la classe d'âge, le statut matrimonial, le statut juridique, l'emploi ou les niveaux de qualification, et enfin selon les contextes sociaux. L'inscription quasi-territorialisée des filles (les représentations sociales renvoient à l'espace des quartiers) concerne moins leurs mères, plutôt assignées à l'espace de la maison, hormis lorsqu'elles tiennent le rôle de « médiatrices culturelles ». Dans l'espace professionnel par contre, ces filles et femmes pourront être regardées différemment selon le niveau de qualification des emplois considérés. Elles sont en général plus distinctement identifiées par leur origine nationale, là où l'on s'attend à trouver des femmes d'" origine immigrée » à savoir dans les emplois faiblement qualifiés, que dans les emplois qualifiés, les postes à responsabilité, notamment dans les corps de métier peu féminisés. En revanche, leur activité professionnelle d'enseignante les amène souvent à leur corps défendant, à tenir un rôle informel de médiatrices culturelles entre le personnel d'encadrement et les élèves «issus de l'immigration" dans le règlement des conflits notamment. Dans les quartiers, elles sont aussi sollicitées comme "passeuses" ou médiatrices. Bien souvent, l'image des enseignantes est ramenée, malgré elles, à l'histoire de leurs élèves et à l'histoire familiale qui est censée leur être commune. Or, il est bon de rappeler que les parcours de ces femmes comme de ces filles présentent de multiples facettes et que toutes les familles d'origine maghrébine ne sont pas toutes sur le même modèle comme peut encore le laisser à penser une certaine imagerie sociale: familles nombreuses, garçons occupant la position d'aînés dans les fratries, pères chefs de famille, etc. Enfin, ces regards portés sur les femmes et les hommes issus de l'immigration maghrébine, d'après nos observations, sont bien sexués : on cherchera, par exemple, à atténuer le rappel des « origines » chez les femmes occupant des emplois peu qualifiés à la différence de leurs homologues masculins. En outre, la pratique consistant à solliciter les enseignants pour leur faire jouer un rôle de médiateur culturel informel semble toucher plus fréquemment les femmes que les hommes. 


\section{BIBLIOGRAPHIE}

ANDEZIAN Sossie, STREIFF-FÉNART Jocelyne (1978) Transformation des rôles traditionnels chez les femmes immigrées, Pluriel, 14, pp. 53-62.

ASSOULINE Florence (1992) Musulmane une chance pour l'Islam, Paris, Flammarion, 223 p.

AYAT Fatima (1990) Les rites de ritualisation du corps de deux générations de femmes marocaines à Rabat. Thèse de $3^{\text {ème }}$ cycle en Sociologie. Sous la dir de J-M Berthelot, Université Toulouse le Mirail, Toulouse.

AYAT-KFITA Najat (1988) Les rites de mariage à travers deux générations de femmes kénitriennes. Enquête sur quelques aspects du changement social au Maroc. Thèse de $3^{\text {ème }}$ cycle en Sociologie. Sous la dir. de J-M Berthelot, Université Toulouse le Mirail, Toulouse.

BELHADJ Marnia (1998) Une révolution tranquille : stratégies professionnelles et dynamique familiale d'un groupe de femmes françaises d'origine algérienne. Thèse de $3^{\text {ème }}$ cycle en Sociologie. Sous la dir. de D. Schnapper. EHESS, Paris.

BILLION Pierre (2001) Les travailleurs sociaux dits « issus de l'immigration », Informations sociales, 113, pp. 112-123.

BOUKHOBZA Noria (2002) Les femmes dans l'ombre du jour. Une histoire d'une famille entre la France et l'Algérie, Cahors, L'Hydre, 168 p.

BOUKHOBZA Noria (2005) Jeux et enjeux d'appropriation des espaces collectifs par une population d'origine maghrébine, in Sociétés de voisins. Vivre en habitat collectif, sous la direction de A. Morel et B. Haumont, Paris, Éditions de la Maison des Sciences de l'Homme, Cahiers d'ethnologie de la France, 21, sous presse.

BOUKHOBZA Noria (2001) Dénouer les noces, Terrain, 36, pp. 45-56.

BOUKHOBZA Noria, DELAVAULT Huguette, HERMANN Claudine (2002) Les enseignantes-chercheuses à l'université. Demain la parité ? Paris, L'Harmattan, 192 p.

BOULAHBEL Yeza (1991) Le secret des femmes; Stratégies familiales et stratégies d'émigration des femmes algériennes. Thèse de Doctorat d'État en Sociologie, sous la direction de D. Schnapper, EHESS, Paris, $475 \mathrm{p}$.

DAUNE-RICHARD Anne-Marie, FLAMENT Catherine, LEMAIRE Madeleine et MARRY Catherine (1986) Les filles dans les formations industrielles de technicien supérieur liées aux nouvelles technologiesle cas de BTS et de DUT dans l'académie d'Aix-Marseille, Rapport de recherche, Aix-en-Provence, LEST-CNRS.

DRAOUA Faouzia (1987) L'étudiante algéroise face au mariage. L'épanouissement personnel compromis, l'épanouissement personnel par le compromis. Thèse de $3^{\text {ème }}$ cycle en Sociologie. Sous la dir. de C. Camilleri. Paris V.

GASPARD Françoise (1995) De l'invisibilité des migrantes et de leurs filles à leur instrumentalisation, Migrants-Formation, $\mathrm{n}^{\circ} 105$, pp. 15-30.

GASPARD Françoise (1994) Le statut personnel des résidents étrangers et le statut personnel et l'intégration sociale, culturelle et nationale. Rapport au Conseil national des populations immigrées. 
GASPARD Françoise, KHOSROKHAVAR Farhad (1994) La problématique de l'exclusion. De la relation des garçons et des filles de culture musulmane dans les quartiers défavorisés. Revue française des Affaires sociales, $\mathrm{n}^{\circ}$ 2, pp. 3-26.

GUENIF-SOUILAMAS Nacira, MACÉ Éric (2004) Les féministes et le garçon arabe, Paris, éditions de l'Aube, $106 \mathrm{p}$.

HASSINI Mohamed (1997) L'école, une chance pour les filles de parents maghrébins. Paris, L'Harmattan, $272 \mathrm{p}$.

JAZOULI Adil (1988) L'intégration au féminin. Les jeunes filles d'origine maghrébine en France : de la famille à la société. Presse et immigrés en France, 169-170, pp. 1-30.

MOROKVASIC Mirjana (1983) Émigration féminine et femmes immigrées : discussion de quelques tendances dans la recherche. Pluriel, 36, pp. 20-51.

NINI Soraya (1993) Ils disent que je suis une beurette, Paris, Fixot, $282 \mathrm{p}$.

POTTIER Céline (1993) La fabrication sociale de médiateurs culturels : le cas de jeunes filles d'origine maghrébine, Revue Européenne des Migrations Internationales, IX (3), pp. 177-190.

QUIMINAL Catherine (2000) Le mouvement des « sans papières », Cahiers du CEDREF, pp. 380-401.

RAHAL-SIDHOUM Saïda (1996), Au cœur des passions, Confluences Méditerranée, n 19, automne.

SAYAD Abdelmalek (1992) Les maux à mots de l'immigration, Migrants-Formation, 90, pp. 34-44.

ZOIA Geneviève, MIGNARD Jean-François (1994) Légitimités des médiations : Deux modèles associatifs et deux territoires toulousains de développement social des quartiers. Rapport de recherche.

Programme : Le lien social dans les périphéries urbaines. Ministère de la culture et de la communication, mission du patrimoine ethnologique, juillet.

VASSAS Claudine (2004) Accords et désaccords, Terrain, 42, pp. 5-16.

\section{NOTES}

1. D'autres émeutes suivront : Vaux en Velin (1990), Toulouse Le Mirail et Montauban (1999).

2. Fatima Ayat, Najat Kfita, Farida Draoua se sont plus particulièrement interrogées sur le mariage. Yeza Boulahbel, Marnia Belhadj ont traité des stratégies migratoires et de l'insertion professionnelle des femmes.

3. Le terme renvoie au viol collectif.

4. Voir aussi les travaux menés par Daune-Richard, Flament, Lemaire et Marry, (1986) sur des étudiantes en BTS industriel. Deux cas de figures se présentent. Le premier introduit la trajectoire des mères «qui considèrent que leurs ambitions scolaires et professionnelles ont été empêchées ou limitées du fait de leur position de sexe dans la famille ». Le second cas de figure renvoie aux «mères qui transmettent un message à leurs filles et ces dernières choisissent explicitement une filière masculine par défi par rapport à ce qui leur est apparu comme une exclusion-interdiction du fait de leur sexe ».

5. Cf L'insertion professionnelle des jeunes diplômés issus de l'immigration: synthèse du diagnostic d'aide à la décision de la direction de l'AFIJ, réalisé avec le soutien du FAS, 1999.

6. Certificat d'Aptitude au Professorat de l'Enseignement Technique.

7. Certificat d'Aptitude au Professorat de l'Enseignement Secondaire.

8. Cette action a été menée conjointement avec l'association Intermed' et les éducateurs du quartier d'Empalot de Toulouse pendant trois ans. 
INDEX

Mots-clés : relations sociales, relations entre les sexes, représentations, femmes, Maghrébins Index géographique : France

\section{AUTEUR}

NORIA BOUKHOBZA

Enseignante chargée de l'égalité des chances à l'IUFM (Institut Universitaire de Formation des Maîtres) de Midi-Pyrénées, chercheuse au Centre d'Anthropologie (EHESS-CNRS),

noria.boukhobza@toulouse.iufm.fr 